\title{
Millimeter dust continuum emission revealing the true mass of giant molecular clouds in the Small Magellanic Cloud ${ }^{\star}$
}

\author{
C. Bot ${ }^{1,2}$, F. Boulanger ${ }^{2}$, M. Rubio ${ }^{3}$, and F. Rantakyro ${ }^{4}$ \\ 1 UMR7550, Centre de Données Astronomiques de Strasbourg (CDS), Université Louis Pasteur, 67000 Strasbourg, France \\ e-mail: bot@astro.u-strasbg.fr \\ 2 Institut d'Astrophysique Spatiale, Université Paris-Sud, 91405 Orsay, France \\ 3 Departamento de Astronomia, Universidad de Chile, Casilla 36-D, Santiago, Chile \\ ${ }^{4}$ European Southern Observatory, Casilla 19001, Santiago 19, Chile
}

Received 20 October 2006 / Accepted 3 May 2007

\section{ABSTRACT}

\begin{abstract}
Context. CO observations have been the best way so far to trace molecular gas in external galaxies, but in low metallicity environments the gas mass deduced could be largely underestimated due to enhanced photodissociation of the $\mathrm{CO}$ molecule. Large envelopes of $\mathrm{H}_{2}$ could therefore be missed by $\mathrm{CO}$ observations.

Aims. At present, the kinematic information of $\mathrm{CO}$ data cubes are used to estimate virial masses and trace the total mass of the molecular clouds. Millimeter dust emission can also be used as a dense gas tracer and could unveil $\mathrm{H}_{2}$ envelopes lacking CO. These different tracers must be compared in different environments.

Methods. This study compares virial masses to masses deduced from millimeter emission, in two GMC samples: the local molecular clouds in our Galaxy $\left(10^{4}-10^{5} M_{\odot}\right)$, and their equivalents in the Small Magellanic Cloud (SMC), one of the nearest low metallicity dwarf galaxies.

Results. In our Galaxy, mass estimates deduced from millimeter (FIRAS) emission are consistent with masses deduced from gamma ray analysis and therefore trace the total mass of the clouds. Virial masses are systematically larger (twice on average) than mass estimates from millimeter dust emission. This difference decreases toward high masses and has been reported in previous studies. This is not the case for SMC giant molecular clouds: molecular cloud masses deduced from SIMBA millimeter observations are systematically higher (twice on average for conservative values of the dust to gas ratio and dust emissivity) than the virial masses from SEST CO observations. The observed excess cannot be accounted for by any plausible change of dust properties. Taking a general form for the virial theorem, we show that a magnetic field strength of $\sim 15 \mu \mathrm{G}$ in SMC clouds could provide additional support for the clouds and explain the difference observed.

Conclusions. We conclude that masses of SMC molecular clouds have so far been underestimated. Magnetic pressure may contribute significantly to their support.
\end{abstract}

Key words. ISM: clouds - submillimeter - ISM: molecules - Magellanic Clouds - ISM: magnetic fields

\section{Introduction}

Molecular clouds are of particular importance as the sites of star formation in a galaxy. Most of this gas is located in large complexes known as giant molecular clouds (GMCs), but the exact amount of gas contained remains unknown since $\mathrm{H}_{2}$ is almost impossible to observe directly in cold interstellar regions where molecules form and survive.

Carbon monoxide (CO) has played a major role in GMC studies and is still the most widely used tracer of molecular gas. However, it may be present only in the densest parts of molecular clouds, leaving $\mathrm{H}_{2}$ envelopes unseen. This effect has been clearly observed in Molecular Clouds of the Solar Neighbourhood (Grenier et al. 2005) and tends to underestimate molecular cloud masses estimated from CO observations. This bias is most important in low metallicity environments where

* The SIMBA data for the SMC is available in electronic form at the CDS via anonymous ftp to cdsarc.u-strasbg.fr (130.79.128.5) or via

http://cdsweb.u-strasbg.fr/cgi-bin/qcat?J/A+A/471/103 the reduced shielding causes $\mathrm{CO}$ to be confined to tiny dense structures (André et al. 2004). Most of the mass may then lie in the cloud envelopes where the gas is molecular due to $\mathrm{H}_{2}$ selfshielding but CO is photodissociated (Israel 2005; Lequeux et al. 1994). Millimeter dust emission could bring to light the total mass of such clouds.

The advent of sensitive bolometer arrays on submillimeter/millimeter telescopes makes long wavelength dust emission an alternative to $\mathrm{CO}$ to compute molecular masses. The good correlation with $\mathrm{CO}$ emission in $\mathrm{CO}$ bright galaxies (e.g. NGC 891 Guelin et al. 1993; NGC 6946 Alton et al. 2002) supports its association with molecular gas. More generally, existing observations show that sub-millimeter dust emission from galaxies traces the total hydrogen column density (Thomas et al. 2002; Meijerink et al. 2005). It complements the $\mathrm{HI}$ and $\mathrm{CO}$ observations to provide a complete view of cold interstellar matter.

We propose to use dust continuum emission at millimeter wavelengths to compute molecular cloud masses in two samples of GMCs: molecular clouds in the Solar neighbourhood, 

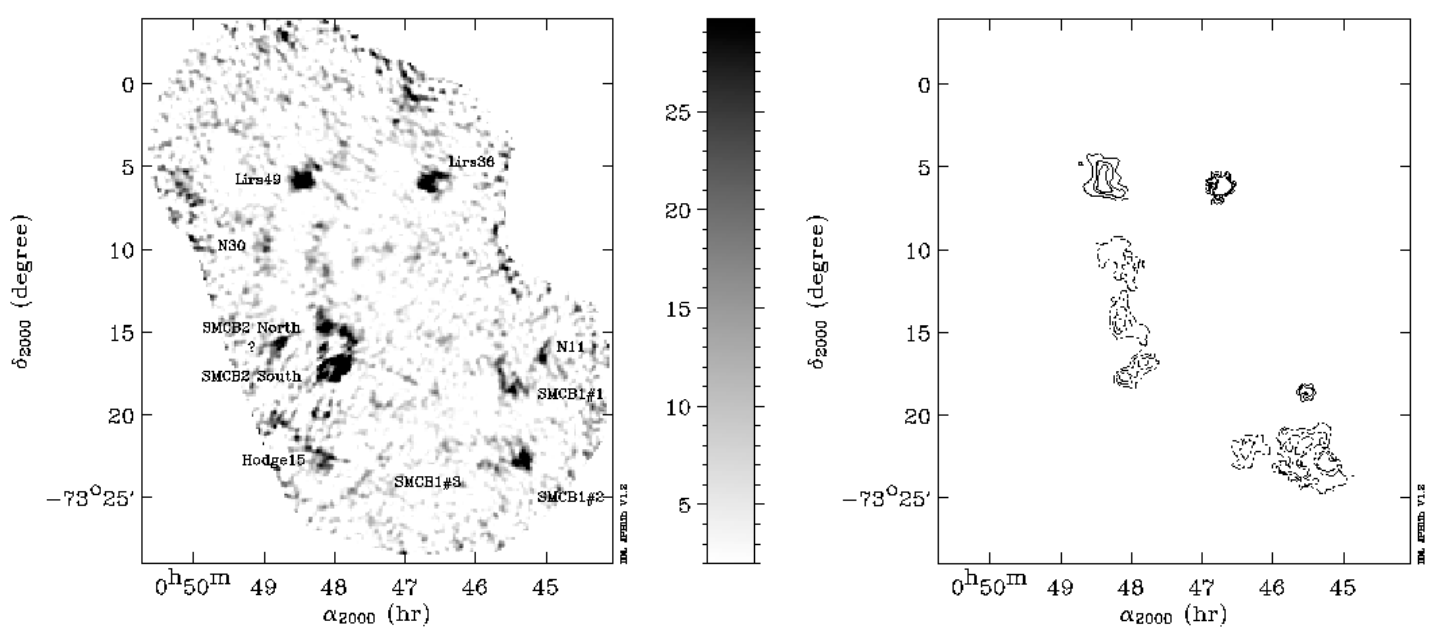

Fig. 1. SIMBA mosaic of the south of the SMC (left panel). Star forming regions studied in this paper are labeled. In the right panel, CO contours of the studied regions are displayed on the same grid (with plain lines when $\mathrm{CO}(2-1)$ is available, with mixed lines for $\mathrm{CO}(1-0)$ data).

and counterparts in the Small Magellanic Cloud (SMC). The SMC is a nearby irregular dwarf galaxy $(D \approx 61 \mathrm{kpc}$; Laney $\&$ Stobie 1994) known to have a low metallicity $\left(Z \approx Z_{\odot} / 10\right.$, Dufour 1984) and giant star forming regions. While the amount of molecular material observed via $\mathrm{CO}$ emission is low, numerous young stars are observed. Furthermore, galaxy counts behind the SMC imply extinctions much larger than expected from the column density of atomic hydrogen (Lequeux 1994). Thus the $\mathrm{SMC}$ is a good candidate to detect extended envelopes of $\mathrm{H}_{2}$ gas through dust observations.

From the comparison of GMCs $\mathrm{CO}$ luminosity and virial mass, the SMC $X_{\mathrm{CO}}=N\left(\mathrm{H}_{2}\right) / I_{\mathrm{CO}}$ conversion factor is estimated to be 10 times the Galactic value (Rubio et al. 1993a; Mizuno et al. 2001). This virial calibration of $X_{\mathrm{CO}}$ should take into account the cloud mass in the $\mathrm{CO}$-poor $\mathrm{H}_{2}$ gas, but dust observations question this view. Lisenfeld et al. (2002) inferred a $X_{\mathrm{CO}}$ conversion factor 25 to 30 times higher than the Galactic value by comparing sub-millimeter dust and $\mathrm{CO}$ emission from the low metallicity dwarf galaxy NGC 1569. Rubio et al. (2004) reported the detection of millimeter dust emission from SMCB1\#1 with SIMBA/SEST. They converted the flux into a cloud mass estimate that is one order of magnitude larger than its $\mathrm{CO}$ virial mass. Leroy et al. (2007) analysed Spitzer far infrared maps in the SMC, in conjunction with $\mathrm{HI}$ and $\mathrm{CO}$ observations. Comparing the $160 \mu \mathrm{m}$ map to NANTEN CO observations they estimate $X_{\mathrm{CO}}$ to be 60 times the Galactic value. Our study continues the work of Rubio et al. (2004) by analysing a larger number of molecular clouds in the SMC and by analysing these clouds together with a reference sample that we built in our Galaxy. As such it is the first comprehensive comparison of two giant molecular cloud mass estimates at different metallicities: gas masses from dust millimeter emission and virial masses. Are the dust and virial mass estimates in agreement? If they are discrepant, how can the difference be explained?

After an overview of the data used in this study (Sect. 2), molecular gas masses from dust millimeter emission are computed for a sample of Local GMCs and for detected SMC GMCs (Sect. 3.1). A comparison to virial mass estimates is performed in Sect. 3.2 and the differences are interpreted in Sect. 4

\section{The data}

This study follows the detection of several molecular clouds at $1.2 \mathrm{~mm}$ in the SMC with the SIMBA bolometer. We present these new observations in detail, along with previously published data.

\subsection{SEST observations in the SMC}

\subsubsection{CO observations}

The CO observations of the SMC clouds were observed as part of the ESO/SEST Key Programme on CO in the Magellanic Clouds (Israel et al. 1993; Rubio et al. 1993a,b, 1996; Israel et al. 2003). The motivation for undertaking these observations in the transitions $J=(1-0)$ and $J=(2-1)$ of the CO molecule and the reduction treatments are described in these papers. The beam has a $F W H M$ of $43^{\prime \prime}$ and $22^{\prime \prime}$ at the $\mathrm{CO}(1-0)$ and the $\mathrm{CO}(2-1)$ frequencies respectively. Since $\mathrm{CO}(2-1)$ line data has better resolution, gas masses computed for the $\mathrm{CO}(2-1)$ emitting regions will be favored when $\mathrm{CO}(2-1)$ observations were performed. In the following, we use the spatial (radius) and kinematic (velocity dispersion) characteristics of the clouds that were computed in the litterature.

\subsubsection{SIMBA millimeter continuum observations}

The SIMBA bolometer was mounted on the Swedish ESO Submillimeter Telescope (SEST). It operated at a central frequency of $250 \mathrm{GHz}(1.2 \mathrm{~mm})$ with a $F W H M$ bandwidth of $90 \mathrm{GHz}$, and an angular resolution of $24^{\prime \prime}$ on the sky. The individual maps were produced using the fast scanning mode. The elimination of the correlated sky noise, adding of the individual maps, and the photometry were performed using the MOPSI package ${ }^{1}$. The $\tau_{\text {zenith }}$ was determined performing skydips on average $2 \mathrm{~h}$ during the day, and every $3 \mathrm{~h}$ during the night. The flux determination was done for each individual observing run by taking maps of Uranus. The data reduction was performed as described in the SIMBA User manual ${ }^{2}$. The flux measurements in the final coadded map have an uncertainty of $15 \%$.

The millimeter dust emission in the SMC is faint and the regions unambigously detected are giant molecular clouds (GMCs) (see Fig. 1). Most of them have been observed in $\mathrm{CO}$ and are named according to Rubio et al. (1993a,b) or

1 MOPSI is a data reduction software package for IR and radio data developped by R. Zylka, IRAM, Grenoble, France.

${ }^{2}$ http://puppis.ls.eso.org/staff/simba/manual/simba/ 
Table 1. Observed characteristics of the detected GMCs in the SMC.

\begin{tabular}{lll}
\hline \hline Name & $\begin{array}{l}\text { Area } \\
\mathrm{pc}^{2}\end{array}$ & $\begin{array}{l}S_{1.2 \mathrm{~mm}}{ }^{a} \\
\mathrm{mJy}\end{array}$ \\
\hline LIRS49 & 357 & $181 \pm 55$ \\
LIRS36 & 385 & $184 \pm 48$ \\
SMCB1\#1 & 108 & $47 \pm 7$ \\
SMCB1\#2 & 260 & $119 \pm 51$ \\
SMCB1\#3 & 38 & $5.8 \pm 0.7$ \\
Hodge15 & 103 & $40.4 \pm 2.5$ \\
SMCB2 S & 695 & $398 \pm 158$ \\
SMCB2 N & 314 & $132 \pm 46$ \\
N11 & 87 & $34.5 \pm 5$ \\
\hline
\end{tabular}

a Corrected for the contribution of free-free emission and the $\mathrm{CO}(2-1)$ line.

Henize (1956). SIMBA fluxes for each region are displayed in Table 1. They are measured for a SIMBA beam convolved to $27^{\prime \prime}$ and within the $25 \mathrm{mJy} /$ beam contour, after subtraction of the flux measured in an empty zone.

Detected sources: Detected GMCs are often associated with well-known H II regions and young clusters and all but LIRS49 correspond to absorption patches (Hodge 1974). We review here some of their properties:

- LIRS49 and LIRS36 are two of the most prominent SMC sources in the far-infrared (Schwering \& Israel 1989) and a large variety of molecular species have been observed in these directions (Chin et al. 1998; Heikkila et al. 1998). LIRS49 may be a superposition of two H II regions belonging to different kinematical complexes along the line of sight (Rosado et al. 1994) or it could be associated with a bubble or a loop (Meyssonnier \& Azzopardi 1993).

- SMCB1\#1 and Hodge15 are two quiescent molecular clouds (not associated with $\mathrm{H} \alpha$ ). The SIMBA millimeter observations of SMCB1\#1 were used by Rubio et al. (2004) to deduce the cloud mass.

- SMCB1\#2 and SMCB1\#3 are parts of the SMC-B1 region of Rubio et al. (1993b) and are associated with large H II regions.

- The SMCB2 region contains several molecular clouds observed by Rubio et al. (1993a). Since these clouds are difficult to separate in the continuum dust emission which has no velocity information, they were grouped into two complexes: SMCB2 North (corresponds to SMCB2\#3 and \#4 of Rubio et al. (1993a)) and SMCB2 South (which corresponds to SMCB2\#1, \#2 and \#6). The SMCB2\#5 region of Rubio et al. (1993a) is not detected at $1.2 \mathrm{~mm}$ with SIMBA.

- Millimeter emission is also detected toward the N30 and N11 (Henize 1956) H II regions, but these regions have not yet been mapped in $\mathrm{CO}$, so no mass comparison can be done for these objects. For N30, the SIMBA flux can be accounted for entirely by free-free emission.

- The source identified by the question mark in Fig. 1 (between SMCB2 North and South) does not correspond to any presently known source. It is clearly detected at the $6 \sigma$ level but there is no associated infrared or radio component. It should be noted however that it lies on an extended component seen at $70 \mu \mathrm{m}$ with Spitzer observations and it is close to the DEM S43 H II region. Further observations are needed to understand the nature of this source.

The observed regions correspond to different evolutionary stages of molecular clouds - from quiescent clouds to evolved massive star forming regions - enabling us to compare $\mathrm{CO}$ and dust mass estimates in different physical conditions.

Origin of millimeter continuum emission: The emission measured at $1.2 \mathrm{~mm}$ consists of several components: thermal dust emission, free-free radiation and ${ }^{12} \mathrm{CO}(2-1)$ line emission. Since our interest is only in cold dust emission, we estimate the other contributions that have to be removed.

CO line emission: The ${ }^{12} \mathrm{CO}(2-1)$ line contribution to the SIMBA surface brightness can be estimated as follows:

$F_{\text {line }}=\frac{2 k v^{3} c^{-3}}{\Delta v_{\text {bol }}} \Omega I_{\mathrm{CO}(2-1)}$,

where $I_{\mathrm{CO}(2-1)}$ is the intensity of the ${ }^{12} \mathrm{CO}(2-1)$ line in $\mathrm{K} \mathrm{km} \mathrm{s}^{-1}$, $\Delta v_{\text {bol }}$ is the bandwidth of the SIMBA bolometer $(90 \mathrm{GHz}), \Omega$ is the region of integration, $k$ is the Boltzmann constant and $v=$ $230 \mathrm{GHz}$. For regions where $\mathrm{CO}(2-1)$ (Rubio et al. 1993b,a, 1996; Israel et al. 2003) has been observed, the total flux density due to line contributions was evaluated (see Table 1) and was found to be negligible.

Free-free emission: The contribution of the continuum emission due to thermal electrons can be estimated with radio continuum data, assuming pure free-free emission at high frequencies and extrapolating this frequency dependence to $1.2 \mathrm{~mm}$ $(250 \mathrm{GHz})^{3}$. We also assume that radio emission is optically thin at long frequencies. We used the ATCA continuum cata$\log$ of the SMC radio sources (Payne et al. 2004) at 1.42, 2.37, 4.8 and $8.64 \mathrm{GHz}$. In both SIMBA and ATCA observations, extended emission on scales larger than a given threshold is filtered out. For SIMBA, extended emission on scales larger than $\sim 2^{\prime}$ is filtered out when subtracting the sky emission. The interferometric ATCA observations are not corrected for missing short spacing and do not measure emission on spatial scales larger than about half the size of the primary lobe. At $8.64 \mathrm{GHz}$, the two angular scales are comparable. Radio emission comes both from free-free and synchrotron emission but at these high frequencies it is dominated by free-free. We estimate the free-free contribution by extrapolating the $8.64 \mathrm{GHz}$ radio flux when available, since it is the less synchrotron biased frequency of this catalog and also the resolution is the closest to the SIMBA one. When no $8.64 \mathrm{GHz}$ flux is relevant, we take the longest frequency available and follow the same procedure: for LIRS49, the free-free contribution is extrapolated from the $2.37 \mathrm{GHz}$ flux; for SMCB2 North and South region it is obtained from the $4.8 \mathrm{GHz}$ fluxes. The free-free contribution could be overestimated In these cases, a bias that would reinforce the result (see Sect. 4).

Dust emission associated with neutral atomic gas HI. In the SMC, the resolution of HI observations does not allow an estimate of the contribution of the SIMBA flux that is associated with neutral gas. However, most of this emission is spatially extended and filtered out in the subtraction of the sky emission.

For each source, dust emission is the major component of the $1.2 \mathrm{~mm}$ flux. In the following, we refer to the SIMBA flux tabulated in Table 1 as the flux measured at $1.2 \mathrm{~mm}$ subtracted from the gas contribution estimates.

\footnotetext{
${ }^{3}$ For electron temperatures typical of HII regions $\left(\sim 10^{4} \mathrm{~K}\right)$, the freefree emission scales as $(0.95-0.16 \ln v)$ where $v$ is in $\mathrm{GHz}$ (Reynolds 1992).
} 
Table 2. Observed characteristics of the Galactic GMCs studied.

\begin{tabular}{lllllll}
\hline \hline Name & $l_{\min }, l_{\max } / b_{\min }, b_{\max }$ & $\begin{array}{l}\text { Distance } \\
\mathrm{pc}\end{array}$ & $\begin{array}{l}S_{1.2 \mathrm{~mm}}{ }^{a} \\
\mathrm{mJy}\end{array}$ & $\begin{array}{l}T_{\text {dust }} \\
\mathrm{K}\end{array}$ & $\begin{array}{l}R \\
\mathrm{pc}\end{array}$ & $\begin{array}{l}\Delta V \\
\mathrm{~km} \mathrm{~s}^{-1}\end{array}$ \\
\hline Orion a & $204.6,216.4 /-23.9,-13.6$ & 450 & $11000 \pm 3000$ & 19.1 & 28.9 & 8.0 \\
Orion b & $202.1,205.9 /-14.4,-11.1$ & 450 & $1700 \pm 300$ & 19.1 & 11.6 & 3.6 \\
Taurus & $170.1,188.9 /-19.9,-10.1$ & 140 & $14000 \pm 4000$ & 16.2 & 13.5 & 3.8 \\
Ophiucus & $347.1,359.9 / 11.6,24.9$ & 130 & $15000 \pm 4000$ & 19.6 & 11.5 & 3.8 \\
Cepheus & $102.1,116.9 / 12.1,20.9$ & 300 & $9700 \pm 4000$ & 16.1 & 22.5 & 6.8 \\
Chamaeleon & $292.1,305.9 /-19.9,-10.6$ & 160 & $6100 \pm 3000$ & 14.8 & 6.2 & 4.6 \\
Lupus & $330.1,344.9 / 10.1,19.9$ & 140 & $7000 \pm 4000$ & 17.4 & 5.2 & 5.1 \\
Perseus & $156.6,161.9 /-22.9,-15.1$ & 320 & $4700 \pm 1500$ & 17.1 & 14.6 & 6.9 \\
\hline
\end{tabular}

${ }^{a}$ Corrected for the contribution of dust associated with $\mathrm{H} \mathrm{I}$.

\subsection{FIRAS and CO data in our Galaxy}

To complement the analysis of SMC clouds, we use FIRAS spectra and the $\operatorname{CO}(1-0)$ sky survey from Dame et al. (2001) for a sample of Local GMCs defined in rectangular $(l, b)$ boxes ${ }^{4}$ : Orion, Taurus, Ophiuchus, Cepheus, Chamaeleon, Lupus and Perseus. The $7^{\circ}$ FIRAS beam for these clouds at a few hundred parsecs and the SIMBA beam at the distance of the SMC correspond to similar linear spatial resolutions.

\subsubsection{Millimeter fluxes}

The $1.2 \mathrm{~mm}$ fluxes were derived by fitting the FIRAS spectra at $\lambda>500 \mu \mathrm{m}$ with a black body multiplied by a power law emissivity. The temperatures in Table 2 were derived from a single temperature fit of the full FIRAS spectra down to $150 \mu \mathrm{m}$ and an emissivity index of 2 . As for the SIMBA observations, the $1.2 \mathrm{~mm}$ emission observed toward the Galactic molecular clouds originates from components other than cold dust emission. We estimate their contribution to the $1.2 \mathrm{~mm}$ flux.

The $C O(2-1)$ line emission contribution is difficult to estimate without $\mathrm{CO}(2-1)$ data. However, since brightness temperatures are the same in $\mathrm{CO} J=2-1$ and $J=1-0$ lines, the Dame et al. (2001) survey can be used to estimate this contribution. Using a $I_{\mathrm{CO}(J=2-1)} / I_{\mathrm{CO}(J=1-0)}=0.6$ for the Solar Neighborhood (Hasegawa 1996), this contribution is found to be negligeable.

Free-free emission: extinction corrected-H $\alpha$ emission (Dickinson et al. 2003) is used to estimate the free-free emission contribution to the FIRAS fluxes. This is found to be very low $(<3 \%)$ and is therefore negligeable.

Dust emission associated with neutral atomic gas. Unlike the SIMBA observations, the FIRAS data measures the total Galactic emission. Part of this emission comes from dust associated with the diffuse medium. We used the Leiden/Argentine/Bonn (LAB) HI survey of Kalberla et al. (2005) to estimate the proportion of millimeter emission associated with neutral gas. At high galactic latitude, the dust millimeter emission is well correlated with HI column densities (Boulanger et al. 1996). The dust emission per $\mathrm{H}$ atom is combined with the HI column densities measured in the cloud areas to compute and subtract the $1.2 \mathrm{~mm}$ flux associated with neutral gas. Their contribution amounts to about $40 \%$ of the total FIRAS flux at $1.2 \mathrm{~mm}$. The $1.2 \mathrm{~mm}$ fluxes obtained are listed in Table 2 for each cloud. To estimate the uncertainties associated with the dust emission removal, we defined for each cloud a reference area of similar size in the symetric position within the Galaxy (same longitude, opposite latitude) and subtract the flux observed in the reference regions from the one observed in

\footnotetext{
4 The box were defined on the millimeter emission map.
}

the molecular clouds. The differences observed are similar to the uncertainties on the data quoted in Table 2

\subsubsection{CO emission}

We use the $\mathrm{CO}(1-0)$ survey of Dame et al. (2001) of the Galaxy to compute the size and velocity dispersion of each cloud over the same areas as the millimeter emission observations. To be consistent with CO information in the SMC, we chose to compute the size and the velocity dispersion of each cloud in a similar way (e.g. no correction for velocity gradients). For each cloud, we fit each spectrum above a certain level and sum the Gaussian fits to derive a total cloud spectrum. The velocity dispersion of the cloud is determined by fitting a Gaussian to the resulting spectrum. This method is close to what was used for the SMC except that some SMC clouds were divided into substructures when independent clouds were separated in spatialvelocity space. We checked that this difference in method for some clouds does not induce a significant bias in the cloud parameters. The radius and velocity dispersions are listed in Table 2 for each cloud. Because there is insufficient spatial resolution to correct for velocity gradients in the SMC clouds, we chose not to correct for them in the Galactic sample either. We are careful to apply the same method to deduce virial masses in both data sets.

\section{Estimates of molecular cloud masses}

\subsection{Molecular gas mass from millimeter continuum emission}

Dust millimeter continuum emission is associated with molecular gas. In this section, we compute molecular cloud masses from the dust emission at $1.2 \mathrm{~mm}$.

The far-infrared/millimeter emission from dust can be expressed as:

$I_{\lambda}=N_{\mathrm{H}} \epsilon_{\mathrm{H}}(\lambda) B_{\lambda}\left(T_{\text {dust }}\right)$

where $N_{\mathrm{H}}$ is the hydrogen column density of the cloud, $\epsilon_{\mathrm{H}}(\lambda)$ is the emissivity of dust per hydrogen atom at the wavelength $\lambda$ and $T_{\text {dust }}$ is the dust temperature. The emissivity per hydrogen atom can be expressed in terms of the absorption coefficient per unit dust mass $\kappa(\lambda)$ :

$\epsilon_{\mathrm{H}}(\lambda)=\kappa(\lambda) x_{\mathrm{d}} \mu m_{\mathrm{H}}$

where $x_{\mathrm{d}}$ is the dust-to-gas mass ratio, and $\mu \mathrm{m}_{\mathrm{H}}$ is the gas weight per $\mathrm{H}$, taking into account the contribution of He. This emissivity may be determined empirically from the correlation between dust and gas observations, independently of any dust model (e.g. 
Boulanger et al. 1996, for the diffuse interstellar medium in the Solar Neighborhood).

While dust temperatures for the Galactic clouds have been deduced from the FIRAS spectra fitting, this information is not available for the clouds in the SMC. Since we wish to apply the same method for both samples, we will fix the dust temperature for both samples. Within each cloud, dust is present over a range of temperatures. The fit of FIRAS spectra with a single temperature gives higher weight to the warmer dust present at the cloud surface or near embedded stars. But in a molecular cloud, the bulk of the mass is shielded from UV radiation. The dust temperature in the shielded parts of the clouds is observed to be smaller than the values in Table 2. For example, based on submillimeter observations, Stepnik et al. (2003) report a dust temperature of $12 \mathrm{~K}$ for a Taurus filament. To conservatively estimate cloud masses, we adopt a dust temperature of $15 \mathrm{~K}$ (the minimum value of the FIRAS temperatures listed in Table 2) to derive Galactic and SMC cloud masses from their millimeter dust fluxes.

We assume that the dust grain opacity per unit mass is the same for the SMC and Milky Way molecular clouds. Therefore, the emissivity per hydrogen atom scales as the dust-to-gas mass ratio. Knowing the SMC dust-to-gas mass ratio and the Galactic dust emissivity per hydrogen atom in the SMC, the millimeter emission can therefore be translated into a gas amount. The Galactic emissivity per hydrogen atom is adopted for dust associated with molecular gas (see Appendix A): $\epsilon_{\mathrm{H}}^{\text {ref }}\left(1.2 \mathrm{~mm}, \mathrm{H}_{2}\right)=$ $(1.2 \pm 0.2) \times 10^{-26} \mathrm{at}^{-1} \mathrm{~cm}^{2}$. For the SMC, this emissivity will be reduced by one sixth to account for the difference in dust-to-gas ratio with respect to the Solar Neighbourhood (see Appendix B). The values of dust emissivity per hydrogen atom that we use correspond to the absorption coefficient per unit mass $\kappa=0.72 \mathrm{~cm}^{2} \mathrm{~g}^{-1}, 3$ times higher than the standard value from Draine \& Lee (1984). Furthermore, the dust-to-gas ratio in the SMC could be as much as 5 times smaller (Bot et al. 2004). The mass estimates we deduce from millimeter emission in the SMC are therefore upper limits. These choices were made to emphasize the robustness of the results of this study, but the range of parameters available for the dust emissivity and dust-to-gas ratio is assessed throughout the paper (see Sect. 4 and Fig. 2).

We can thus translate the measured millimeter fluxes of the clouds into their corresponding hydrogen column densities and molecular cloud masses. The results for the Solar Neighborhood and the SMC clouds are presented in Tables 3 and 4 respectively. Note that we used an emissivity per unit dust mass and a dustto-gas ratio that are 1.8 and 1.7 times higher, respectively, than the one used by Rubio et al. (2004).

\subsection{Comparison to virial masses}

In both data sets, the kinematic and spatial information provided by the $\mathrm{CO}$ data cube enables the determination of virial masses. For this determination, we use the simplified formulation for a $1 / r$ density law from MacLaren et al. (1988):

$M_{\mathrm{vir}}\left(M_{\odot}\right)=190 \Delta V^{2}\left(\mathrm{~km} \mathrm{~s}^{-1}\right) R(\mathrm{pc})=\frac{9 \sigma^{2} R}{2 G}$

where $\Delta V$ is the observed velocity dispersion from the CO linewidth (FWHM) and $R$ is the radius of the cloud. The virial masses for both cloud samples are computed using the radius and velocity dispersion of the clouds. For the SMC, these parameters were taken from the litterature (cf. Sect. 2.1.1). For the Galactic clouds, the radius and velocity dispersion were computed as described in Sect. 2.2.2, in order to be consistent with

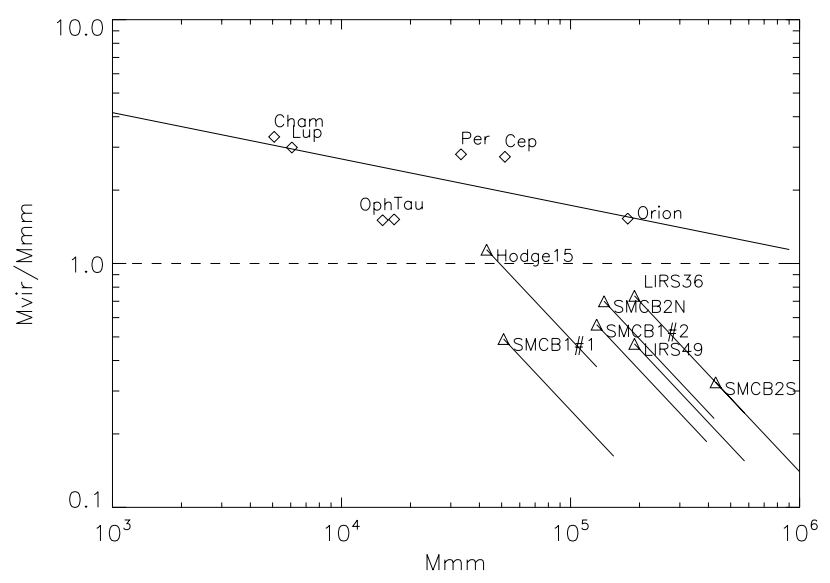

Fig. 2. Evolution of the ratio of the virial mass and the mass deduced from millimeter emission with respect to the mass deduced from the millimeter dust emission in solar masses $M_{\odot}$. The Galactic clouds are represented by diamonds while the SMC clouds are shown with triangles. The law of Solomon et al. (1987) is also plotted (the solid line), representing the evolution of a larger sample of Galactic clouds. For the SMC clouds, the uncertainties on masses deduced from millimeter emission (regarding the assumptions on the dust to gas ratio and the dust emissivity) are represented by a straight line for each cloud.

Table 3. Different mass estimates of the Galactic GMCs studied.

\begin{tabular}{|c|c|c|c|}
\hline Name & $\begin{array}{l}M_{\mathrm{H}}^{\mathrm{mm}} \\
10^{4} M_{\odot}\end{array}$ & $\begin{array}{l}M_{\mathrm{H}}^{\text {vir }} \\
10^{4} M_{\odot}\end{array}$ & $f=\frac{M_{\mathrm{H}}^{\mathrm{mm}}}{M_{\mathrm{H}}^{\mathrm{vir}}}$ \\
\hline Orion $^{a}$ & $14 \pm 3$ & $27 \pm 2$ & $0.5 \pm 0.2$ \\
\hline Taurus & $1.3 \pm 0.4$ & $2.6 \pm 0.1$ & $0.5 \pm 0.2$ \\
\hline Ophiucus & $1.3 \pm 0.3$ & $2.3 \pm 0.1$ & $0.6 \pm 0.2$ \\
\hline Cepheus & $4.3 \pm 1.8$ & $14.2 \pm 0.8$ & $0.3 \pm 0.2$ \\
\hline Chamaeleon & $0.8 \pm 0.4$ & $1.7 \pm 0.1$ & $0.5 \pm 0.3$ \\
\hline Lupus & $0.7 \pm 0.4$ & $1.8 \pm 0.1$ & $0.4 \pm 0.3$ \\
\hline Perseus & $2.4 \pm 0.7$ & $9.3 \pm 0.4$ & $0.3 \pm 0.1$ \\
\hline
\end{tabular}

${ }^{a}$ The mass are obtained by adding the masses of Orion a and Orion $b$.

Table 4. Different mass estimates of the SMC GMCs studied with the associated uncertainties. Notes: (1) computed from $R$ and $\Delta V$ using CO (1-0) data; (2) computed from $R$ and $\Delta V$ using $\mathrm{CO}(2-1)$ data.

\begin{tabular}{llll}
\hline \hline Name & $\begin{array}{l}M_{\mathrm{H}}^{\mathrm{mm}} \\
10^{4} M_{\odot}\end{array}$ & $\begin{array}{l}M_{\mathrm{H}}^{\text {vir }} \\
10^{4} M_{\odot}\end{array}$ & $f=\frac{M_{\mathrm{H}}^{\mathrm{mm}}}{M_{\mathrm{H}}^{\text {vir }}}$ \\
& $19 \pm 6$ & $8.9 \pm 0.5^{(2)}$ & $2.2 \pm 0.7$ \\
\hline LIRS49 & $19 \pm 5$ & $14.0 \pm 0.5^{(2)}$ & $1.4 \pm 0.4$ \\
LIRS36 & $19 \pm 0.8$ & $2.5 \pm 0.2^{(2)}$ & $2.0 \pm 0.3$ \\
SMCB1\#1 & $5.1 \pm 0.8$ & $7.3 \pm 1.4^{(1)}$ & $1.8 \pm 0.8$ \\
SMCB1\#2 & $13 \pm 6$ & $4.9 \pm 0.4^{(2)}$ & $0.9 \pm 0.1$ \\
Hodge15 & $4.3 \pm 0.3$ & $4.9 \pm 2.3^{(1)}$ & $3.1 \pm 1.2$ \\
SMCB2 S & $43 \pm 17$ & $14.3 \pm 1.9^{(1)}$ & $1.5 \pm 0.5$ \\
SMCB2 N & $14 \pm 5$ & $9.8 \pm 1$. \\
\hline
\end{tabular}

the SMC values. The results for Galactic and SMC clouds are presented in Tables 3 and 4 respectively.

Rosolowsky \& Leroy (2006) have shown that spatial resolution and sensitivity differences in molecular-line data cubes can significantly affect the measured sizes and line widths that are used to deduce virial masses of giant molecular clouds. In the present study, the linear spatial resolution of the SEST $\mathrm{CO}(2-1)$ data in the SMC and of the $\mathrm{CO}(1-0)$ data from Dame et al. (2001) in the solar neighbourhood are similar. Bias 
arising from low resolution should therefore affect the SMC and Galactic sample in the same way. This offset may be more problematic when no $\mathrm{CO}(2-1)$ data was available for some clouds in the $\mathrm{SMC}$ and $\mathrm{CO}(1-0)$ data with larger resolution $(\sim 13 \mathrm{pc})$ was used instead. However, the results we obtain in these cases are not significantly different than the results obtained with higher resolution $\mathrm{CO}$ data so the uncertainties induced do not affect the results of this paper.

Virial masses for the clouds of the Galactic sample have been published previously (de Geus et al. 1990; Ungerechts \& Thaddeus 1987; Boulanger et al. 1998; Grenier et al. 1989). However, the characteristics of the clouds were computed for substructures and the masses of the substructures were then added. We have compared our virial mass estimates with those of the litterature for the same clouds. We observe minor differences that reflect the different definitions in the extent of the clouds and the difference in the method used (velocity gradients, substructure divisions).

\section{Results}

Tables 3 and 4 give mass estimates for the clouds in our Galaxy and in the SMC, computed from millimeter observations of dust emission and from virial estimates. These two estimates of the gas mass of a cloud are compared through the ratio of these masses, $f=\frac{M_{1.2 \mathrm{~mm}}}{M_{\mathrm{vir}}}$.

We observe a systematic difference between the two mass estimates: in our Galaxy, the molecular cloud masses deduced from millimeter emission are systematically lower than the virial masses obtained from CO observations, while in the SMC the mass deduced from millimeter emission are systematically larger. This difference is best seen in Fig. 2.

\subsection{In the Galaxy}

The comparison of the virial mass and the gas mass deduced from millimeter emission in the local Galactic clouds shows that the virial mass is always larger than the mass from millimeter emission, with a mean $f$ factor of 0.5 .

Grenier et al. (2005) analysed $\gamma$ ray emission in Giant molecular clouds of the Solar Neighborhood and found that a significant part of the mass of large molecular clouds is not traced by $\mathrm{CO}$ emission. We compared our mass estimates to their masses of "dark gas" (interpreted as $\mathrm{H}_{2}$ without $\mathrm{CO}$ ) for the same regions. We find that the cloud masses deduced from millimeter dust emission are fully compatible with the total masses of the clouds (the "dark gas" plus molecular gas traced by CO emission). However, for the regions chosen in this study, the fraction of "dark gas" with respect to the total molecular gas is low.

Solomon et al. (1987) compared, for a large sample of giant molecular clouds in our Galaxy, the virial mass of the clouds to their CO luminosity. They observed a good correlation between both quantities that can be transformed into a relation between the mass deduced from CO emission and the virial mass. Since in the studied regions the masses deduced from $\mathrm{CO}$ emission are close to the masses deduced from millimeter dust emission, this law can then be compared to our mass estimates for the Galactic clouds (see Fig. 2). For the Galactic clouds, the millimeter and $\mathrm{CO}$ masses are consistent for the Galactic gamma ray calibration of $X_{\mathrm{CO}}=1.8 \times 10^{20} \mathrm{~mol} \mathrm{~cm}^{-2}\left(\mathrm{~K} \mathrm{~km} \mathrm{~s}^{-1}\right)^{-1}$. For this factor, the Solomon et al. (1987) virial mass- $L_{\mathrm{CO}}$ relation shows that the virial mass is larger than the cloud mass by a factor decreasing toward high masses (see Fig. 2). This trend was also observed for clumps within GMCs by numerous studies in our Galaxy (Bertoldi \& McKee 1992; Williams et al. 1995; Heyer et al. 2001; Simon et al. 2001). In the Galaxy, the $f$ factor is observed to be 1 only for the most massive Galactic GMCs with masses larger than $5 \times 10^{5} M_{\odot}$ (Dame et al. 1986). For clumps within large gravitationally bound clouds the weight of the overlying material in the cloud could provide significant binding pressure for the clumps (Bertoldi \& McKee 1992).

\subsection{In the $S M C$}

The comparison of gas masses obtained from the millimeter dust emission with the virial masses shows a systematic bias in the SMC. Gas masses deduced from the millimeter emission are 1.8 times higher on average than the virial masses. The factor $f=\frac{M_{1.2 \mathrm{~mm}}}{M_{\mathrm{vir}}}$ ranges from 0.9 to 3.1 (see Table 4 ). The mass discrepancy reported for SMCB1\#1 by Rubio et al. (2004) is thus a general result that must be understood.

For the computation of masses from the millimeter flux, we used a dust temperature of $15 \mathrm{~K}$ for all the clouds. Could a higher temperature in the molecular cloud of the SMC explain the difference observed in mass? For the virial mass and the mass from millimeter dust emission to agree, the dust temperature within the molecular gas would have to be $23 \mathrm{~K}$ on average. But if the molecular clouds of the SMC were warm, they would emit in the far infrared. While for most of the regions surveyed here, the Spitzer $160 \mu \mathrm{m}$ emission (Leroy et al. 2007) is biased toward the HII region emission present in the beam, this is not the case for the quiescent molecular cloud SMCB1\#1. Comparing millimeter fluxes with Spitzer data in SMCB1\#1 gives a dust temperature of 11,14 or $17 \mathrm{~K}$ for spectral indices of $2,1.5$ and 1 , respectively. This shows that at least in a quiescent cloud (and presumably in all molecular clouds) a higher dust temperature in the molecular clouds of the SMC is not compatible with the far-infrared data and cannot explain the difference observed between the mass estimates.

Furthermore, the cloud masses deduced from dust millimeter emission depend on the dust-to-gas ratio and the emissivity of dust grains in the SMC molecular clouds. The values we used for these parameters were chosen to limit the mass of the clouds and thus reduce the discrepancy with virial masses. In the diffuse ISM of the SMC, the dust emissivity per hydrogen atom is measured to be $1 / 30$ the emissivity of the Galactic diffuse medium (Bot et al. 2004). For the molecular clouds of the SMC, we used a larger emissivity to account both for grain coagulation effects (twice the diffuse medium value) and for our estimate of the SMC dust-to-gas ratio (twice larger than the canonical value for the metallicity of the SMC, cf. Appendix B). Not only it is not clear if grain coagulation (grain emissivity enhancement by mass unit) occurs in the SMC, but we assume that heavy elements depletions are the same in the SMC and Galactic molecular clouds while they are observed to be smaller in the SMC diffuse ISM. In that sense, we consider the millimeter mass estimates in Table 4 to be lower limits on the molecular cloud masses. Lower depletions and thereby lower dust emissivity per hydrogen atom will enlarge the mass discrepancy. The range of masses obtained for each cloud, for dust emissivities ranging from $1 / 6$ to $1 / 20$ the Galactic molecular value, is shown in Fig. 2. Since it is dynamically difficult to account for a large difference between the cloud mass and its virial estimation, a first conclusion of this paper is that SMC dust observations imply a significant (up to a factor $\sim 5$ ) increase in the dust-to-gas mass ratio from the diffuse ISM to molecular clouds. The dust which is destroyed by shocks 
in the diffuse ISM is rebuilt in molecular clouds. A second conclusion is that even with conservative assumptions on the dust emissivity per hydrogen atom, the virial mass is found to be systematically lower than the millimeter gas mass.

\section{Generalized virial theorem}

In the SMC, molecular cloud masses from millimeter dust emission are systematically larger than the virial masses. We saw above that uncertainties on the mass estimates from dust emission can not explain such differences. Observational estimates of the velocity dispersion are obtained through the $\mathrm{CO}$ line width. The lack of resolution and sensitivity of the observations certainly plays a role in the virial mass computations. However, the observational bias in the Galactic and the SMC sample should be similar and we were careful to apply the same analysis, so we are confident that the virial mass discrepancy in the SMC does not arise from observational uncertainties.

Leroy et al. (2007) studied dust emission in the far-infrared in the SMC and showed that excesses with respect to HI are observed to extend farther than $\mathrm{CO}$ emission observed with NANTEN. This difference is attributed to molecular hydrogen not traced by $\mathrm{CO}$ and amounts to $30 \%$ in spatial coverage. The $\mathrm{CO}$ and SIMBA observations we use for the SMC clouds certainly underestimate the size of the giant molecular clouds due to sensitivity effects. As a result, the masses measured in this study are characteristics of clumps within the clouds smaller than the one from the Leroy et al. (2007) study. However, the size of the clouds that are detected with SIMBA and CO observations (see Fig. 1) in our study are about the same, so that the effect of any biases in the cloud radius should not be large enough to affect our results.

The mass discrepancy observed between the SMC and Galactic clouds might indicate that the $\mathrm{CO}$ line width underestimates the amplitude of the cloud turbulent motion. This interpretation was proposed by Rubio et al. (2004). In the SMC clouds, the $\mathrm{CO}$ emission is expected to come from dense clumps embedded in lower density gas where $\mathrm{CO}$ is photo-dissociated (Lequeux et al. 1994). The bulk of the cloud mass is in the lower density gas but the CO line width only measures the velocity dispersion of the clumps. The same statement applies locally to the less massive high Galactic latitude clouds where $\mathrm{CO}$ line widths are observed to be systematically lower than the HI line width. Quantitatively, in the Ursa Major high latitude cloud, on scales of 5-10 pc, the HI velocity dispersion is observed to be twice that of CO (de Vries et al. 1987). It is not clear whether this observed difference can be simply extrapolated to the more massive SMC clouds that are gravitationally bound. However, it has been theoretically suggested that in gravitationally bound clouds the gas velocity dispersion should increase as the density decreases (Fatuzzo \& Adams 1993; McKee 1999). In this section, we present an alternative interpretation where the mass difference results from the contribution of the magnetic field to the cloud support.

In this section, we inspect the assumptions made for the virial mass calculations, searching for those which could cause a systematic underestimation of the mass. In particular, the presence of a magnetic field in the molecular clouds could help to balance gravity and we will compute the necessary strength to explain the mass ratio $f$ observed in the SMC.

To apply the virial theorem, molecular clouds should be virialized. Observationally determined dynamical parameters enable us to define the characteristic time scale of these clouds: $R / \Delta V$. Clouds younger than this characteristic time did not have time to be virialized and the virial theorem cannot apply. This characteristic time scale for the SMC clouds is $\sim 3 \times 10^{6}$ years. It is therefore possible that these clouds did not have time to be virialized. One possibility would be that in the SMC, unlike Galactic giant molecular clouds, $\mathrm{CO}$ observations only trace clouds collapsing into stars on the timescale of a few million years (free-fall time). But this interpretation does not fit with the fact that two clouds, SMCB1\#1 and Hodge15, have no associated HII.

In this study, virial masses were determined using the simplified form from MacLaren et al. (1988). This form neglects among others the surface pressure and the magnetic field terms. The general virial equilibrium equation for a cloud in a steady state can be written as:

$0=2\left(\mathcal{T}-\mathcal{T}_{0}\right)+\mathcal{M}+\mathcal{W}$

(McKee \& Zweibel 1992) where $\mathcal{T}$ is the total kinetic energy (due to thermal and global motions), $\mathcal{T}_{0}$ represents the confinement by external thermal pressure, $\mathcal{M}$ is the magnetic energy and $\mathcal{W}$ is the gravitational energy. The gravitational energy can be expressed as:

$W=-\frac{3}{5} a \frac{G M^{2}}{R}$

where $G$ is the gravitational constant and $a$ measure departures from uniform density and sphericity (Bertoldi \& McKee 1992).

McKee \& Zweibel (1992) introduced the virial parameter $\alpha=a \frac{2 \mathcal{T}}{|\mathcal{W}|}$ so that the mass of a cloud is expressed as:

$M=\frac{5 \sigma^{2} R}{\alpha G}$

If we take $M_{1.2} \mathrm{~mm}$ as the true gas mass of the cloud, understanding the high values of $f=M_{1.2} \mathrm{~mm} / M_{\text {vir }}$ amounts to looking for low values of the virial parameters (with $f=10 / 9 \alpha$ ), which is equivalent to solving:

$f=\frac{10}{9 a} \frac{\left[1-\left(P_{0} / \bar{P}\right)\right]}{[1-(\mathcal{M} /|\mathcal{W}|)]}$

The values of $\mathrm{f}$ are then affected by density and geometry effects $(a)$, external pressure $\left(P_{0}\right)$ and magnetic fields $(\mathcal{M} /|\mathcal{W}|)$. As we saw in Galactic giant molecular clouds, $f$ is smaller than unity and is interpreted as external pressure confinement. Since external pressure (high $P_{0}$ ) acts in lowering $f$ and the effects of density and geometry are mild, we will only discuss the effects of magnetic field support in the SMC giant molecular clouds.

\subsection{Magnetic field support in clouds.}

From the observed characteristics of the molecular clouds, we can compute the magnetic-to-gravitational energy ratio needed to explain the $f$ factors. Taking the case of a spherical uniform cloud with steady motion and a mean pressure exceeding the surface pressure $\left(\bar{P} \gg P_{0}\right)$, one obtains $\mathcal{M} /|\mathcal{W}|=1-\frac{10}{9 f}$. The obtained values are displayed in Table 5 .

Taking $\mathcal{M}=\frac{1}{6}\left(B^{2}-B_{0}^{2}\right) R^{3}$, one can express the strength of the magnetic field that is necessary to account for the $f$ values:

$B^{2}-B_{0}^{2}=\frac{18}{5}\left(1-\frac{10}{9 f}\right) \frac{G M^{2}}{R^{4}}$ 
Table 5. Deduced characteristics for the SMC clouds.

\begin{tabular}{lllll}
\hline \hline Name & $f$ & $\mathcal{M} /|\mathcal{W}|$ & $|B|$ & $\begin{array}{l}n_{\mathrm{H}} \\
\mu \mathrm{G}\end{array}$ \\
& & & $10^{3} \mathrm{~cm}^{-3}$ \\
\hline LIRS 49 & 2.2 & 0.5 & 24 & 0.8 \\
LIRS 36 & 1.4 & 0.2 & 9 & 0.3 \\
SMCB1\#1 & 2.0 & 0.5 & 12 & 0.5 \\
SMCB1\#2 & 1.8 & 0.4 & 11 & 0.3 \\
Hodge 15 & 0.9 & - & - & 0.4 \\
SMCB2 S & 3.1 & 0.6 & 20 & 0.3 \\
SMCB2 N & 1.5 & 0.2 & 11 & 0.4 \\
\hline
\end{tabular}

where $B_{0}$ is the ambient medium value of the magnetic field strength. Taking $B_{0}=5 \mu \mathrm{G}^{5}$, the magnetic field strength necessary to support the clouds is computed and displayed in Table 5. They range from $\sim 10$ to $\sim 30 \mu \mathrm{G}$, with a mean value of $\approx 15 \mu \mathrm{G}$. Such strengths are similar to the values reported for some Galactic clouds (Myers \& Goodman 1988) but the clumps surveyed are much smaller and confined by the pressure, not by their self-gravity (Bertoldi \& McKee 1992). Recently, a magnetic field with a similar strength $(\sim 20 \mu \mathrm{G})$ has been observed in the outer part of the Taurus molecular cloud (Wolleben \& Reich 2004).

Thus, magnetic field support of the molecular clouds could explain the mass difference observed in the SMC. But why would the magnetic field support be important for the SMC molecular clouds and not for the Galactic ones? Molecular cloud formation could be different in the SMC. Giant molecular clouds in spiral galaxies are thought to form in spiral arms where the low shear and high gas density allows gravity to gather diffuse matter over large scales. Matter may preferentially condense along field lines without compressing the magnetic field. We refer to Rosolowsky et al. (2003) for a discussion of observational constraints on proposed formation scenarios based on a survey of molecular clouds in M 33. In an irregular galaxy like the SMC, massive stars have an impact on the structure of the interstellar medium and molecular clouds may form out of shells blown by stellar winds and supernovae explosions. In the Spitzer infrared images (Bolatto et al. 2007), the molecular clouds in Fig. 1 appear as local peaks along a large shell of 300 pc diameter. In this picture, the magnetic field is expected to be swept together with the matter. It is also possible that the lower metallicity and therefore the lower dust content in the SMC creates lower dust opacity in the GMCs of the SMC than in our Galaxy. The ionization could therefore be higher in the SMC, supporting higher magnetic fields. Another possibility is that magnetic field support is important also in the Galactic molecular clouds and virial masses could have to be re-examined taking into account magnetic field support on large scales. In this case, it would be interesting to see if the larger external pressure that will be required for the virial mass to match the other mass estimates (from $\gamma$ ray analysis for example) is compatible with observations. The magnetic field observations (e.g. through the Zeeman effect) can be used to confirm the magnetic field strength in these regions.

\section{Conclusions}

We compared two different molecular gas tracers, the millimeter dust emission and the virial theorem applied to $\mathrm{CO}$ observations, at two different metallicities: our Galaxy and the SMC.

\footnotetext{
5 Consistent with the Pohl (1993) model; note that this choice does not influence the result as long as the mean SMC magnetic field remains low, which we have no reason to doubt.
}

SIMBA/SEST millimeter observations for the SMC and FIRAS observations for the Galaxy have a similar linear resolution, enabling a uniform study similar giant molecular clouds in these two environments.

We find a clear difference between the two samples: in the Galaxy, the virial mass is systematically larger than the mass deduced from millimeter emission, while in the SMC sample, the virial mass is lower than the mass deduced from millimeter emission. The main uncertainty in the mass determination comes from the dust emissivity per hydrogen atom in the SMC. We have used the millimeter emissivity determined in Galactic molecular gas scaled by $1 / 6$ to account for the lower SMC metallicity. This scaling factor has been calculated asssuming similar depletions of heavy elements in the SMC and in the Galaxy. We consider this assumption to be conservative because diffuse ISM depletions have been inferred to be smaller in the SMC than in the Galaxy from UV spectroscopy and dust observations. The millimeter cloud masses would have been larger for smaller depletions (i.e. smaller dust-to-gas ratio), enhancing the discrepancy.

For Galactic clouds the dust and $\mathrm{CO}$ masses are consistent with the gamma ray detemination of the Galactic $X_{\mathrm{CO}}$ factor. The virial masses are higher than the cloud masses. The underestimation of SMC molecular cloud masses by virial methods is thus an unexpected result. Inspecting the assumptions made in the virial mass computations, we find that magnetic field support in the clouds could explain the difference between the two mass estimates. In this scenario, SMC giant molecular clouds are not fully supported by turbulent motion. Additional support is given by a magnetic field of $\sim 15 \mu \mathrm{G}$. Further studies and magnetic field observations are needed to confirm this interpretation.

An alternative explanation is that the virial mass is underestimated because the $\mathrm{CO}$ line width does not measure the full amplitude of the turbulent motion within the cloud. In the SMC GMCs, the CO emission is expected to come from dense clumps embedded in lower density gas where $\mathrm{CO}$ is photo-dissociated. The bulk of the cloud mass is in the lower density gas, but the CO line width only measures the velocity dispersion of the clumps. Can the lower density gas turbulence be higher than the clump to clump velocity dispersion? This study emphasizes the power of millimeter emission as a dense matter tracer. The large amount of millimeter data soon to be available will enable interesting follow up studies with better resolution and sensitivity.

Acknowledgements. We thank the anonymous referee for useful comments, T. Dame for providing us with the CO Galactic survey data cube and I. Grenier for helping with the comparison with the gamma ray results. This study has been supported but the french national program PCMI (Physique et Chimie du Milieu Interstellaire, CNRS). M.R. is supported by the Chilean Center for Atrophysics FONDAP No. 15010003. C.B. and F.B. wish to aknowledge the Chilean Center for Astrophysics FONDAP No 150010003 for support of their visit in Chile.

\section{Appendix A: Emissivity of dust associated with molecular gas}

Dust emissivity per hydrogen atom is often measured in the diffuse medium where it is easy to deduce directly from observations. However, dust properties could vary from diffuse to dense regions (Cambrésy et al. 2001; Stepnik et al. 2003; Cambrésy et al. 2005). We therefore chose to recompute the emissivity of dust in the molecular ring in order to represent the properties of dust associated with molecular hydrogen.

In the molecular ring, the $1.2 \mathrm{~mm}$ dust continuum and $\mathrm{CO}$ emission longitude profiles along the Galactic plane are 
well correlated and the ratio of their intensities is constant: $R=(0.89 \pm 0.01) \times 10^{-1} \mathrm{MJy}\left(\mathrm{K} \mathrm{km} \mathrm{s}^{-1} \mathrm{sr}\right)^{-1}$ (Boulanger et al., in prep.). This ratio is related to the well known CO-to- $\mathrm{H}_{2}$ conversion factor $\left(X=N\left(\mathrm{H}_{2}\right) / I_{\mathrm{CO}}\right)$ :

$R=2 \epsilon_{\mathrm{H}}(\lambda) B_{\lambda}\left(T_{\text {dust }}\right) X$,

where $\epsilon_{\mathrm{H}}$ is the dust emissivity per hydrogen atom in the observed region. Although $T_{\text {dust }}$ may vary from one cloud to the other, these variations are likely to be moderate and can therefore be neglected at millimeter wavelength while $T>10 \mathrm{~K}$. In this study, we consider the temperature range measured by FIRAS as pertinent for molecular cloud analysis. The CO-to- $\mathrm{H}_{2}$ factor is empirically determined in different environments and is discussed in the literature.

In our Galaxy and in particular in the solar neighbourhood, studies of $\gamma$ rays and gas tracers (Strong et al. 1988) enable a determination of this $X$ factor. For our Galaxy as a whole, the analysis of recent observations gives $X$ factors between 1.56 and $1.9 \times 10^{20} \mathrm{~mol} \mathrm{~cm}^{-2}\left(\mathrm{~K} \mathrm{~km} \mathrm{~s}^{-1}\right)^{-1}$ (Strong \& Mattox 1996; Hunter et al. 1997; Grenier et al. 2005). We use the recent estimate obtained for the local molecular clouds through $\gamma$ ray analysis (Grenier et al., priv. comm.): $X=1.8 \pm 0.3 \times$ $10^{20} \mathrm{~mol} \mathrm{~cm}^{-2}\left(\mathrm{~K} \mathrm{~km} \mathrm{~s}^{-1}\right)^{-1}$.

Using this local Galactic $X$ factor and using the ratio of millimeter and $\mathrm{CO}$ intensities determined in the molecular ring, we compute the emissivity of dust associated with molecular gas in our Galaxy:

$\epsilon_{\mathrm{H}}^{\mathrm{ref}}\left(1.2 \mathrm{~mm}, \mathrm{H}_{2}\right)=(1.20 \pm 0.20) \times 10^{-26} \mathrm{at}^{-1} \mathrm{~cm}^{2}$.

This value is used in Eq. (2) to compute molecular cloud masses.

The dust emissivity in the diffuse medium is measured through the correlation at high Galactic latitude between dust emission and $\mathrm{H}$ I emission at low column densities (Boulanger et al. 1996). For a dust temperature of $17.2 \mathrm{~K}$, this correlation gives a dust emissivity per hydrogen atom of:

$\epsilon_{\mathrm{H}}^{\mathrm{ref}}(1.2 \mathrm{~mm}, \mathrm{HI})=(6.7 \pm 1.2) \times 10^{-27} \mathrm{at}^{-1} \mathrm{~cm}^{2}$.

Dust emissivity in the molecular medium is therefore twice that in the diffuse medium. Such an increase in emissivity from the diffuse medium to molecular clouds was observed in our Galaxy, but in much smaller and denser regions (Cambrésy et al. 2001; Stepnik et al. 2003). This increase is observed to be correlated with a drop in small grain abundance. It has been thus interpreted as the coagulation of small grains between themselves or on large grains in dense regions of molecular clouds, giving composite porous grains with a larger emissivity.

\section{Appendix B: The dust to gas ratio in the SMC}

The gas-to-dust ratio in the SMC has been determined by using extinction curves to derive $N(\mathrm{H} \mathrm{I}) / A(\mathrm{~V})$ values (Gordon et al. 2003; Bouchet et al. 1985; Tumlinson et al. 2002). However, the derived values correspond to specific line of sights (where extinction can be measured), they rely on the present extinction law understanding, and there is a significant scatter from one region to the other and from one study to the other ${ }^{6}$. Bot et al. (2004) deduced a gas-to-dust ratio 30 times the solar value by comparing the dust and H I emission of the SMC. However, this value

6 The dust to has ratio found this way to range between $\sim 1 / 6$ and $\sim 1 / 30$ the Galactic value. refers to the diffuse medium and could reflect dust grain destruction processes that do not apply so intensively in the denser environments of the SMC bar where grains are more shielded. A generally used value for the SMC dust-to-gas ratio is one tenth of the solar one, using the metallicity difference (Dufour 1984). However, it relates to gas phase abundances while we are interested in dust masses and, most importantly, since 1984 the solar abundances have been updated (Sofia \& Meyer 2001b,a).

We therefore chose to recompute the SMC dust abundances to evaluate the dust-to-gas mass ratio difference between the SMC and the Solar Neighbourhood. For that, we assume that the elemental depletions are the same in the SMC as in the Solar Neighbourhood. This is supported by studies of Sk 108 in the main bar of the SMC, where the depletion patterns are similar to those of Galactic halo clouds (Welty et al. 1997; Mallouris et al. 2001). The depletions in the SMC could be less than the solar values (Rolleston et al. 2003), so that our estimation could be an upper limit. Comparing the $\zeta$ Oph dust and solar abundances given by Sofia \& Meyer (2001b,a), we inferred depletions $\eta$ of $0.66,0.11,0.46,0.92$, and 0.95 for $\mathrm{C}, \mathrm{N}, \mathrm{O}, \mathrm{Mg}$, and $\mathrm{Si}$ respectively.

The main sequence star AV 304 shows no sign of processing in its photosphere and could therefore represent the present-day total (gas and dust) composition of the SMC (Rolleston et al. 2003). Using Trundle et al. (2004) corrected abundances for this star with the depletion factors computed above, the dustto-hydrogen mass ratio can be computed:

$\frac{m_{\mathrm{dust}}}{m_{\mathrm{H}}}=\sum_{\mathrm{M}}[\mathrm{M} / \mathrm{H}]_{\mathrm{tot}} \cdot \eta_{\mathrm{M}} \cdot \frac{m_{\mathrm{M}}}{m_{\mathrm{H}}}$,

and is used as an estimate of the dust-to-gas mass ratio. We obtained a dust-to-hydrogen mass ratio of $1.51 \times 10^{-3}$ for the SMC. Comparing this value to the dust-to-hydrogen mass ratio for $\zeta \mathrm{Oph} \mathrm{dust}^{7}$, the dust-to-gas mass ratio difference between the SMC and the Solar Neighbourhood is found to be:

$\frac{x_{\mathrm{SMC}}}{x_{\odot}}=0.17$.

\section{References}

Alton, P. B., Bianchi, S., Richer, J., Pierce-Price, D., \& Combes, F. 2002, A\&A, 388,446

André, M. K., Le Petit, F., Sonnentrucker, P., et al. 2004, A\&A, 422, 483

Bertoldi, F., \& McKee, C. F. 1992, ApJ, 395, 140

Bolatto, A. D., Simon, J. D., Stanimirović, S., et al. 2007, ApJ, 655, 212

Bot, C., Boulanger, F., Lagache, G., Cambrésy, L., \& Egret, D. 2004, A\&A, 423, 567

Bouchet, P., Lequeux, J., Maurice, E., Prevot, L., \& Prevot-Burnichon, M. L. 1985, A\&A, 149, 330

Boulanger, F., Abergel, A., Bernard, J.-P., et al. 1996, A\&A, 312, 256

Boulanger, F., Bronfman, L., Dame, T. M., \& Thaddeus, P. 1998, A\&A, 332, 273

Cambrésy, L., Boulanger, F., Lagache, G., \& Stepnik, B. 2001, A\&A, 375, 999

Cambrésy, L., Jarrett, T. H., \& Beichman, C. A. 2005, A\&A, 435, 131

Chin, Y.-N., Henkel, C., Millar, T. J., Whiteoak, J. B., \& Marx-Zimmer, M. 1998, A\&A, 330, 901

Dame, T. M., Elmegreen, B. G., Cohen, R. S., \& Thaddeus, P. 1986, ApJ, 305, 892

Dame, T. M., Hartmann, D., \& Thaddeus, P. 2001, ApJ, 547, 792

de Geus, E. J., Bronfman, L., \& Thaddeus, P. 1990, A\&A, 231, 137

de Vries, H. W., Thaddeus, P., \& Heithausen, A. 1987, ApJ, 319, 723

Dickinson, C., Davies, R. D., \& Davis, R. J. 2003, MNRAS, 341, 369

Draine, B. T., \& Lee, H. M. 1984, ApJ, 285, 89

Dufour, R. J. 1984, in Structure and Evolution of the Magellanic Clouds, IAU Symp., 108, 353

Fatuzzo, M., \& Adams, F. C. 1993, ApJ, 412, 146

\footnotetext{
$7 m_{\text {dust }} / m_{\mathrm{H}}(\zeta \mathrm{Oph})=8.88 \times 10^{-3}$ as computed from Sofia \& Meyer (2001b,a) abundance values.
} 
Gordon, K. D., Clayton, G. C., Misselt, K. A., Landolt, A. U., \& Wolff, M. J. 2003, ApJ, 594, 279

Grenier, I. A., Lebrun, F., Arnaud, M., Dame, T. M., \& Thaddeus, P. 1989, ApJ, 347,231

Grenier, I. A., Casandjian, J., \& Terrier, R. 2005, Science, 307, 1292

Guelin, M., Zylka, R., Mezger, P. G., et al. 1993, A\&A, 279, L37

Hasegawa, T. 1996, in CO: Twenty-Five Years of Millimeter-Wave Spectroscopy, ed. W. B. Latter, J. E. Radford Simon, P. R. Jewell, J. G. Mangum, \& J. Bally, IAU Symp., 170, 39

Heikkila, A., Johansson, L. E. B., \& Olofsson, H. 1998, A\&A, 332, 493 Henize, K. G. 1956, ApJS, 2, 315

Heyer, M. H., Carpenter, J. M., \& Snell, R. L. 2001, ApJ, 551, 852

Hodge, P. W. 1974, PASP, 86, 263

Hunter, S. D., Bertsch, D. L., Catelli, J. R., et al. 1997, ApJ, 481, 205

Israel, F. P. 2005, A\&A, 438, 855

Israel, F. P., Johansson, L. E. B., Lequeux, J., et al. 1993, A\&A, 276, 25

Israel, F. P., Johansson, L. E. B., Rubio, M., et al. 2003, A\&A, 406, 817

Kalberla, P. M. W., Burton, W. B., Hartmann, D., et al. 2005, A\&A, 440, 775

Laney, C. D., \& Stobie, R. S. 1994, MNRAS, 266, 441

Lequeux, J. 1994, A\&A, 287, 368

Lequeux, J., Le Bourlot, J., Des Forets, G. P., et al. 1994, A\&A, 292, 371

Leroy, A., Bolatto, A. D., Stanimirović, S., et al. 2007, ApJ, 658, 1027

Lisenfeld, U., Israel, F. P., Stil, J. M., \& Sievers, A. 2002, A\&A, 382, 860

MacLaren, I., Richardson, K. M., \& Wolfendale, A. W. 1988, ApJ, 333, 821

Mallouris, C., Welty, D. E., York, D. G., et al. 2001, ApJ, 558, 133

McKee, C. F. 1999, in The Origin of Stars and Planetary Systems, ed. C. J. Lada, \& N. D. Kylafis, NATO ASIC Proc., 540, 29

McKee, C. F., \& Zweibel, E. G. 1992, ApJ, 399, 551

Meijerink, R., Tilanus, R. P. J., Dullemond, C. P., Israel, F. P., \& van der Werf, P. P. 2005, A\&A, 430, 427

Meyssonnier, N., \& Azzopardi, M. 1993, A\&AS, 102, 451
Mizuno, N., Rubio, M., Mizuno, A., et al. 2001, PASJ, 53, L45

Myers, P. C., \& Goodman, A. A. 1988, ApJ, 326, L27

Payne, J. L., Filipović, M. D., Reid, W., et al. 2004, MNRAS, 408

Pohl, M. 1993, A\&A, 279, L17

Reynolds, R. J. 1992, ApJ, 392, L35

Rolleston, W. R. J., Venn, K., Tolstoy, E., \& Dufton, P. L. 2003, A\&A, 400, 21

Rosado, M., Le Coarer, E., \& Georgelin, Y. P. 1994, A\&A, 286, 231

Rosolowsky, E., Engargiola, G., Plambeck, R., \& Blitz, L. 2003, ApJ, 599, 258

Rosolowsky, E., \& Leroy, A. 2006, PASP, 118, 590

Rubio, M., Lequeux, J., \& Boulanger, F. 1993a, A\&A, 271, 9

Rubio, M., Lequeux, J., Boulanger, F., et al. 1993b, A\&A, 271, 1

Rubio, M., Lequeux, J., Boulanger, F., et al. 1996, A\&AS, 118, 263

Rubio, M., Boulanger, F., Rantakyro, F., \& Contursi, A. 2004, A\&A, 425, L1

Schwering, P. B. W., \& Israel, F. P. 1989, A\&AS, 79, 79

Simon, R., Jackson, J. M., Clemens, D. P., Bania, T. M., \& Heyer, M. H. 2001, ApJ, 551, 747

Sofia, U. J., \& Meyer, D. M. 2001a, ApJ, 558, L147

Sofia, U. J., \& Meyer, D. M. 2001b, ApJ, 554, L221

Solomon, P. M., Rivolo, A. R., Barrett, J., \& Yahil, A. 1987, ApJ, 319, 730

Stepnik, B., Abergel, A., Bernard, J.-P., et al. 2003, A\&A, 398, 551

Strong, A. W., \& Mattox, J. R. 1996, A\&A, 308, L21

Strong, A. W., Bloemen, J. B. G. M., Dame, T. M., et al. 1988, A\&A, 207, 1

Thomas, H. C., Dunne, L., Clemens, M. S., et al. 2002, MNRAS, 331, 853

Trundle, C., Lennon, D. J., Puls, J., \& Dufton, P. L. 2004, A\&A, 417, 217

Tumlinson, J., Shull, J. M., Rachford, B. L., et al. 2002, ApJ, 566, 857

Ungerechts, H., \& Thaddeus, P. 1987, ApJS, 63, 645

Welty, D. E., Lauroesch, J. T., Blades, J. C., Hobbs, L. M., \& York, D. G. 1997, ApJ, 489, 672

Williams, J. P., Blitz, L., \& Stark, A. A. 1995, ApJ, 451, 252

Wolleben, M., \& Reich, W. 2004, A\&A, 427, 537 